\title{
Presencia de lesiones en el entrenamiento físico militar. Una experiencia de investigación en la Escuela Militar de Cadetes General José María Córdova*
}

\author{
Laura Elizabeth Castro Jiménez** \\ Paula Janyn Melo Buitrago***
}

Recibido: mayo 21 del 2018 • Aceptado: julio 13 del 2018

\section{Resumen}

El militar que está en proceso de formación es sometido a altas exigencias físicas, mentales y psicológicas que se ven aumentadas por el tiempo de concentración, por las horas de entrenamiento y por la densidad de las actividades. Es por lo anterior, que el proceso de entrenamiento se asemeja al de altos logros por su duración mayor a seis meses de forma consecutiva y diaria, de esta manera el militar presenta un riesgo mayor a la aparición de lesiones, las cuales pueden llevar a procesos de recuperación lentos y generar en algunos casos la deserción de este. El objetivo de la investigación fue conocer las lesiones

"Artículo de investigación, asociado al proyecto de investigación Prevención de lesiones derivadas del entrenamiento físico militar en la Escuela Militar de Cadetes General Cadetes José María Córdova. Fase II - Aplicación. Fase III - Sistema de vigilancia epidemiológica. Citar como: Castro, L. y Melo, P. (2018). Presencia de lesiones en el entrenamiento físico mílitar. Una experiencia de investigación en la Escuela Militar de Cadetes General José María Córdova. Cuerpo, Cultura y Movimiento, 8(2), 35-47. DOI: https://doi.org/10.15332/2422474X.5125

"* Magíster en Salud Pública de la Universidad Nacional, docente investigadora del grupo Cuerpo, Sujeto y Educación de la Facultad de Cultura Física, Deporte y Recreación de la Universidad Santo Tomás, Bogotá D. C., Colombia. Correo electrónico: laura.castro@usantotomas.edu.co / ORCID: https://orcid.org/0000-0001-5166-8084

"*** Magíster en Fisiología del Deporte de la Universidad del Valle, coordinadora de investigaciones de la Facultad de Educación Física Militar en la Escuela Militar de Cadetes General José María Córdova, Bogotá D. C., Colombia. Correo electrónico: paulajanynmb@yahoo.es ORCID: https://orcid.org/0000-0002-3492-7985 
producto del entrenamiento físico militar en la Escuela Militar de Cadetes General José María Córdova. Este estudio fue de tipo cuantitativo, de corte descriptivo. Se diseñó una encuesta para conocer las lesiones producto del entrenamiento físico militar. Dentro de los resultados se evidenció que muchas de las lesiones presentadas son por sobreentrenamiento y sobreesfuerzo por parte de los militares al mando o de los mismos cadetes, motivados por sobresalir, tener mejores condiciones al interior de la Escuela o por cumplir órdenes extras que en su mayoría son ejercicios físicos por acciones en contra de su reglamento. Se determinó que esta es una población que generalmente no es tomada en cuenta en el área de la actividad física y el deporte, por ser la fuerza que protege al país y se desconoce el contexto en que deben entrenarse y las consecuencias de esto para salir a brindar un servicio a la nación.

Palabras clave: alto rendimiento, entrenamiento, militares. 


\section{Presence of injuries Military physical training. Research experience in the Military School of Cadetes General José María Córdova}

Abstract

The military that is in the process of formation is subject to high physical, mental and psychological demands that are increased by the time of concentration, by the hours of training and the density of the activities. It is for the above, that the training process is similar to that of high achievements for its duration greater than six months consecutively and on a daily basis, in this way the military presents a greater risk to the appearance of injuries, which may lead to to slow recovery processes and generate in some cases the desertion of this. The objective of the investigation was to know the injuries resulting from the military physical training in the Military School of General Cadets José María Córdova. This study was of a quantitative type, descriptive in nature. A survey was designed to know the injuries resulting from military physical training. Within the results it was evident that many of the injuries presented are due to overtraining and overexertion on the part of the commanding officers or the cadets themselves, motivated by excelling, having better conditions within the School or by fulfilling extra orders than in their Most are physical exercises for actions against their regulation. It was determined that this is a population that is not generally taken into account in the area of physical activity and sports, as it is the force that protects the country and the context in which they must train and the consequences of this to go out to provide a service to the nation.

Keywords: high performance, training, military. 


\section{Introducción}

Todos los países tienen como base para ejercer su soberanía la conformación de ejércitos, los cuales tienen como objetivo "defender la soberanía, la independencia y la integridad territorial, proteger a la población civil, los recursos privados y estatales para contribuir a generar un ambiente de paz, seguridad y desarrollo, que garantice el orden constitucional de la nación" (Ejército Nacional de Colombia, 2014). Es así, como

[...] la Escuela Militar de Cadetes General José María Córdova, alma mater del Ejército Nacional, es la encargada de formar integralmente a los futuros oficiales del Ejército como líderes comandantes de pelotón, profesionales en ciencias militares y otras disciplinas, con sólidas competencias fundamentales en principios y valores institucionales, en función del desarrollo y la seguridad nacional. (Ejercito Nacional de Colombia, 2014).

De esta manera, la formación académico-militar en Colombia, se da en un tiempo de cuatro años, en los cuales reciben entrenamiento físicomilitar diario y paralelamente cursan estudios universitarios de pregrado en diversas disciplinas como lo son administración logística, ingeniería civil, derecho, relaciones internacionales o educación física militar. Dentro de la formación física militar, se realizan sesiones 4 o 5 días a la semana, con una duración de entre 2 y 3 horas diarias como mínimo, bajo una planificación estructurada de acuerdo al nivel de preparación en el que se encuentran; aspectos que nos permiten considerarlos como deportistas de rendimiento, acercándonos al léxico deportivo, por la elevada exigencia en carga, duración y densidad en el entrenamiento, así como en el aumento de cargas físicas de forma sistemática que se observa en los planes de curso; toda vez que hay que tener en cuenta que el entrenamiento deportivo comprende tres aspectos esenciales como lo son la especificidad, la planificación y el rendimiento (Martin, 2001).

Weineck (2005) define la capacidad de rendimiento deportivo como "el grado de mejora posible de un rendimiento de orden motriz, de una actividad deportiva determinada"; es así como el entrenamiento físico militar, en la búsqueda de lograr el mejor resultado en el campo de batalla, expone a sus hombres a altas exigencias físicas, por las horas de entrenamiento, el tiempo de concentración, por la densidad de las 
actividades y las pruebas de evaluación en las que se miden sus capacidades (Rodríguez, Valenzuela, Velasco, Castro y Melo, 2016). O sea, se puede considerar a esta población como de alto rendimiento, no solo por que duran 6 meses o más con entrenamiento físico militar diario, sino porque con ello persiguen el desarrollo de los potenciales humanos propios de la vida militar desde los aspectos físico, psicológico y social, situación que se acerca a la definición del alto rendimiento deportivo, considerado como "la máxima expresión del desarrollo de los potenciales humanos en una práctica deportiva a máximo nivel competitivo" (Hernández, 2000). Por lo anterior, el entrenamiento físico militar se encuentra asociado inevitablemente con la presencia de lesiones, lo cual en algunos casos conlleva a procesos de rehabilitación lentos que pueden interrumpir la vida militar (OlmedillaZafra, Ortega-Toro, Prieto-Andréu y Redondo, 2009).

Keith, Bruce, Steven y Bullock (2010), en su estudio realizado en los Estados Unidos, mencionan que aunque las lesiones son reconocidas como un importante problema de salud en el Ejército, la magnitud del problema se subestima, cuando solo se consideran las lesiones agudas traumáticas, y los trastornos musculoesqueléticos relacionados con las lesiones son comunes en esta población joven y activa. Muchas de ellas implican daño físico causado por microtraumas (uso excesivo) en la recreación, el deporte, la formación y el desempeño laboral. En 2006, hubo 743,547 lesiones musculoesqueléticas relacionadas (ambulatorias y hospitalarias, combinadas) en militares, incluyendo los diagnósticos primarios y no primarios. En la matriz, el $82 \%$ de los trastornos musculoesqueléticos relacionados con las lesiones se clasificaron en: inflamación/dolor (uso excesivo), seguido de alteraciones articulares $(15 \%)$ y las fracturas por estrés $(2 \%)$. Articulaciones como la rodilla (22\%), la columna lumbar $(20 \%)$, y el tobillo/pie (13\%) son las zonas del cuerpo más lesionadas. Concluyendo, se hace necesario evaluar la magnitud del problema de las lesiones en los servicios militares y reconocer las afecciones musculoesqueléticas e incluirlas. Cuando estas lesiones se combinan con lesiones traumáticas agudas, hay casi 1,6 millones de consultas médicas relacionadas con lesiones cada año (Keith et al., 2010).

Castro-Solano y Casullo (2002) realizaron un estudio que tuvo como objetivo reconocer la asociación entre el rendimiento en los aprendizajes académicos de los militares exitosos y no exitosos, así como en los reclutas 
y los que están próximos a la graduación, teniendo como resultado que los factores relacionados con la preparación físico-militar y sicológicos son poco abordados en esta población. Castro-Solano y Fernandez-Liporace (2005) analizaron seis cohortes de egresados de una institución militar (1997-2003), teniendo en cuenta las variaciones que se presentan en el rendimiento académico, relacionándolo con variables sociodemográficas como nivel educativo y ocupación de padres y estatus de pariente de militar. Los resultados mostraron a nivel académico que los promedios generales presentan una tendencia decreciente en el tiempo; en relación con el rendimiento militar se encontró menor variabilidad, pero existió una correlación estadísticamente significativa entre el rendimiento académico y militar con el nivel educativo parental (Castro-Solano y Fernández-Liporace, 2005).

Mohammadi, Azma, Naseh, Emadifard y Etemadi (2013) mencionan que la alta incidencia de lesiones en los miembros inferiores en reclutas militares asociados con ejercicios físicos sugiere que la fatiga es un factor de riesgo de lesiones; por lo que es necesario evaluar si los ejercicios militares podrían generar adaptaciones en el tobillo y la rodilla y examinar la posible relación entre estas articulaciones en futuras lesiones en el servicio militar. En este mismo estudio se encontró que los militares mostraron desalineación articular después del ejercicio $(\mathrm{p}<0.01)$, pero el grupo de atletas lesionados tenía mayores cambios para todas las variables $(\mathrm{p}<0.01)$.

Con el fin de valorar correctamente la presencia de lesiones osteoartromusculares que se generan por el entrenamiento físico militar, es necesario analizar la prevalencia de estas y los factores asociados a la ocurrencia de las mismas. A tal propósito, cabe señalar que el cotejo estadístico proporciona indicios esenciales para reducir el riesgo y así dar información necesaria para adecuar la carga física y los ejercicios seleccionados para la preparación propicia, de acuerdo con las necesidades del soldado colombiano y disminuir de este modo el peligro de que se presenten condiciones crónicas.

\section{Metodología}

El estudio fue de corte cuantitativo. El diseño de investigación propuesto fue descriptivo, el cual tenía como fin identificar propiedades, características 
y rasgos importantes de cualquier fenómeno que sobresaliera (Hernández Sampiere, 2010).

Se diseñó un formato de recolección de datos, tipo encuesta, dirigido a los cadetes de la Escuela Militar de Cadetes General José María Córdova. A través de este se buscó indagar sobre los siguientes aspectos:

- Presencia de lesiones (número de lesiones).

- Mecanismo de lesión (directo, indirecto, agudo, crónico).

- Tipo de lesión (lesiones osteomusculares).

Después de realizar los ajustes pertinentes al instrumento se aplicó este a los cadetes de la Escuela Militar de Cadetes General José María Córdova. Conseguida la información se almacenó en una base de datos para analizarla a través del programa SPSS. El tamaño de muestra se tomó de una muestra de cadetes de la Escuela Militar de Cadetes General José María Córdova, se decidió que el muestreo se realizara empleando la siguiente fórmula de muestreo:

$$
\mathrm{n}=\frac{\mathrm{N} * \mathrm{Z}^{2}{ }_{\mathrm{a}} p^{*} q}{\mathrm{~d}^{2 *}(\mathrm{~N}-1)+\mathrm{Z}_{\mathrm{a}}^{2}{ }^{*} p^{*} q}
$$

La proporción esperada (p) fue de 0.2 , teniendo en cuenta que actualmente se tiene una población cautiva en la Escuela Militar. Tomar una proporción esperada (p) de 0.2 llevó a la necesidad de ampliar el tamaño de la muestra; el nivel de significancia fue de 0.05 (tomando un valor de confiabilidad del $95 \%$ ).

\section{Resultados y discusión}

Para la descripción de los resultados se menciona lo encontrado en los primeros 5 niveles, ya que a partir del sexto los estudiantes tienen entrenamiento en mando que complejiza aún más el entrenamiento físico militar. De acuerdo a los resultados de las encuestas, se encontró que el $64.6 \%$ de los estudiantes de primer nivel realiza un entrenamiento de 2 horas mínimas de duración, el $27.1 \%$ de 4 horas, y el $8.4 \%$ de más de 5 horas diarias. De 
estos, solo el $10.5 \%$ entrena menos de tres días a la semana, el $35.4 \% 5$ días y el $54.2 \%$ más de 6 días a la semana. Los estudiantes de segundo nivel reportan que el $41.5 \%$ entrena 2 horas diarias, el $32.1 \% 4$ horas y el $22.6 \%$ más de 5 horas; aumentando el porcentaje de entrenamiento de mayor duración con respecto al nivel anterior.

Los de tercer nivel mencionan que el $24.4 \%$ entrena 2 horas diarias, el $45.1 \% 4$ horas y el $26.9 \%$ más de 5 horas diarias; aumentando en comparación a niveles inferiores. En el cuarto nivel, la duración del entrenamiento es de 2 horas en el $50 \%$; 4 horas en el $20.7 \%$ y mayor de 5 horas en el 22.4 $\%$. Con respecto a los de quinto nivel, el $55.6 \%$ tiene un entrenamiento de 2 horas diarias, 4 horas el $25.9 \%$ y $18.5 \%$ más de 5 horas al día. Se encontró un aumento de la duración del entrenamiento cuando se sube el nivel, exceptuando el quinto nivel que disminuyó un poco.

En cuanto a los días de entrenamiento, en el nivel dos se menciona que el $45.3 \%$ entrena 5 días a la semana y el $50.9 \%$ más de 6 días a la semana. El nivel tres refiere que el $57.3 \%$ entrena más de 6 días a la semana, el 23.2 \% 5 días a la semana y el porcentaje restante menos de tres días. Los de cuarto nivel informan que tres días el $24.1 \%$; días el $36.2 \%$ y mayor de 6 días el $36.2 \%$. Y del quinto nivel entrena el $49.4 \% 5$ días a la semana y el $32 \%$ más de 6 días a la semana; mostrándose un mantenimiento en la frecuencia del entrenamiento.

Los cadetes de primer nivel referencian que aparte de su entrenamiento físico militar diario, realizan el promedio en el día de 90.2 repeticiones de flexiones de codo; 64.09 abdominales; 10.39 ejercicios en barra; 2.7 vueltas a la escuela; 2.6 vueltas a "marte" (vuelta a la periferia de la Escuela Militar) y 98.07 sentadillas, por castigos o por órdenes de los militares a cargo. En el segundo nivel el reporte es de 89.05 flexiones de codo; 65.6 abdominales; 15.9 ejercicios con barra; 3 vueltas a la escuela; 3.05 vueltas a marte y 59.5 sentadillas.

Los de tercer nivel mencionan que el promedio de flexiones de pecho son 124.4; el de abdominales de 75.7; ejercicios en barra 15.6; vueltas a la Escuela Militar 3.44; vueltas a marte 2 y 146.5 sentadillas por día. Los de cuarto nivel refieren que de flexiones de codo, el promedio es $73.7 ; 62.4$ abdominales; 9 ejercicios en barra; 1 vuelta a la Escuela Militar y a marte y 76 sentadillas. Los de quinto nivel mencionan que hacen además de su entrenamiento diario, 64.2 flexiones de codo, 63.8 abdominales, 11 ejercicios en barra, 2.9 vueltas a la escuela, 2 vueltas a marte y 47 sentadillas por día. 
Del total de la población de primero a quinto nivel se encontró que el $58.73 \%$ reportó alguna lesión durante su estancia en la Escuela Militar; al discriminar por niveles de formación de los estudiantes los resultados muestran que en primer nivel el $27.78 \%$ de la población se ha lesionado, en segundo y tercer nivel el $30.56 \%$ cada uno, en cuarto nivel el $11.11 \%$ y el $19.44 \%$ en quinto nivel, mostrando una prevalencia elevada. Las lesiones más comunes en orden de porcentaje es la periostitis, seguida del esguince y la tendinitis, y este orden se mantiene en los diferentes niveles evaluados. La mayor parte de las lesiones presentadas en la población es en miembros inferiores, con un porcentaje del $85 \%$.

El entrenamiento de alto rendimiento requiere de una disciplina rigurosa en cuanto a la dedicación en tiempo y al cumplimiento de factores endógenos (constitución corporal, edad...) y exógenos (nutrición, condiciones ambientales...) que influyen definidamente en la obtención de resultados (Heinrich, Spencer, Fehl y Poston, 2012). De la misma manera, el cumplimiento de los principios del entrenamiento deportivo al momento de planificar las cargas es fundamental a la hora de medir la rigurosidad en su ejecución; teniendo en cuenta que para desarrollar la capacidad de rendimiento en un proceso a largo plazo es necesario el planteamiento de objetivos, contenidos, medios y métodos de entrenamiento (Weineck, 2005). El entrenamiento que se desarrolla con los estudiantes de la Escuela Militar de Cadetes, corresponde a un entrenamiento a largo plazo y posee los aspectos necesarios para el desarrollo de la capacidad de rendimiento, ya que posee objetivos claros. El contenido del entrenamiento es propio del contexto militar en cuanto a ejercicios de desarrollo general, específico y competitivo, que en el caso de ellos se realiza cuando ejecutan los controles de condición física a inicio, mediados y finales del semestre y asisten a las campañas en las unidades militares especiales; los medios se manifiestan en todos los escenarios que tienen dispuestos para el desarrollo de sus prácticas militares y los métodos se encuentran en la sistematización y planificación de las cargas de acuerdo al nivel en que se encuentren los estudiantes; de acuerdo a los resultados obtenidos, todo esto puede verse afectado por las cargas adicionales que los estudiantes manifestaron en la encuesta realizada, reflejado en grandes trabajos de fuerza, potencia en brazos y piernas que, según las repeticiones referidas, puede interpretarse en 15 minutos adicionales de trabajo intenso sin ningún tipo de calentamiento ni recuperación, ya que corresponde a los ejercicios improvisados que realizan por orden, $y$ 
la resistencia aeróbica que puede ser de entre 30 minutos y hasta una hora adicional. Todo esto se puede interpretar como características del entrenamiento militar, pero, ¿cuánto de todo esto puede estar afectando el rendimiento óptimo de los estudiantes? La aparición, presencia y permanencia de ciertas lesiones puede ofrecer una mirada diagnóstica y así procurar las soluciones adecuadas.

De acuerdo a lo encontrado, la prevalencia de lesiones de los estudiantes es alta, y esto mismo se ha descubierto en los estudios realizados por Hollingsworts (2009), quien menciona que las lesiones musculoesqueléticas son la principal causa de morbilidad y de ausencia y perdida del entrenamiento en la vida militar; los resultados de este estudio revelan que casi un tercio de todos los miembros del Batallón de Marina de Operaciones Especiales, Delta Company, han experimentado dolor o limitación física debido a una lesión musculoesquelética. De los lesionados, casi el $30 \%$ informó que la lesión afectó su capacidad para entrenar durante su ciclo de entrenamiento predespliegue. Estos resultados confirman que las lesiones músculoesqueléticas son un problema importante en el Comando de Operaciones Especiales del Cuerpo de Marines; la investigación adicional se justifica para examinar los factores etiológicos que resulta en estas lesiones y los cambios en los regímenes de entrenamiento que pueden resultar en disminución en la presencia de lesiones (Hollingsworth, 2009; Martínez, 2011).

Por otra parte, Hong y Chu mencionan que entre abril del 2004 y abril del 2005, los reclutas militares que se quejaban de dolor en las piernas durante las 6 semanas de entrenamiento básico en el grupo de educación y capacitación República de Corea, fueron evaluados de acuerdo a sus manifestaciones clínicas y las radiografías simples, encontrando que 12 de 635 reclutas que se quejaban de dolor de la pierna fueron diagnosticados con fractura por estrés del peroné. Once casos (10 reclutas) aparecieron en la unión de los extremos proximal y medial $1 / 3$ del peroné y 2 casos ( 2 reclutas) estaban en el medio tercio del peroné, según lo evaluado radiológicamente. Todas las fracturas se produjeron después de marchar o saltar en cuclillas, concluyendo que la fractura proximal por estrés del peroné no es rara en los reclutas militares por las actividades propias del entrenamiento físico militar (Hong y Chu, 2009).

Lo anterior muestra que la presencia de lesiones en la población militar es frecuente y lo que se necesita es llamar la atención de los entes encargados 
de su entrenamiento para buscar estrategias que disminuyan la prevalencia de estas y generar, de acuerdo a su finalidad, hombres y mujeres más fuertes que puedan cumplir con la visión del Ejército Nacional, la cual es conformar "soldados multimisión".

\section{Conclusiones}

Los estudiantes de la Escuela Militar por el tiempo de dedicación y de exigencia pueden considerarse como deportistas de alto rendimiento, pero las cargas presentadas tanto físicas como académicas han conllevado a que sean susceptibles de presentar lesiones, las cuales no han sido consideradas por los profesionales del área y que se convierte en la actualidad en una población que ha estado desprotegida frente a los métodos de entrenamiento como a la planificación de estos. Es así como actualmente lo anterior se transforma en un campo de intervención y de cuidado ya que son ellos los que tienen a cargo la seguridad del país y se deben mejorar las condiciones de calidad de vida y de salud de esta población.

\section{Agradecimientos}

A la Escuela Militar de Cadetes General José María Córdova, en especial al decano de la Facultad de Educación Física Militar, mayor Santiago Cortés, por su apoyo en la toma de datos; así como a los estudiantes de semillero HYGEA de la Facultad de Cultura Física, Deporte y Recreación de la Universidad Santo Tomás, Bogotá D. C, Colombia.

\section{Responsabilidad}

La financiación del proyecto estuvo a cargo de la Universidad Santo Tomás y de la Escuela Militar de Cadetes José María Córdova, quienes aportaron con los tiempos de los investigadores para el desarrollo de la investigación. 


\section{Referencias}

Castro-Solano, A. y Casullo, M. (2002). Predictores del rendimiento académico y militar de cadetes argentinos. Anales de Psicología/Annals of Psychology, 18(2), 247-259. Recuperado de https://revistas.um.es/analesps/article/ view/28451/27551

Castro-Solano, A. y Fernández-Liporace, M. (2005). Predictores para la selección de cadetes en instituciones militares. Psykhe (Santiago), 14(1), 17-30. DOI: https://doi.org/10.4067/s0718-22282005000100002.

Ejército Nacional de Colombia. (2014). Ejército Nacional de Colombia. Misión y Visión. Recuperado de http://www.ejercito.mil.co/?idcategoria=362168

Heinrich, K., Spencer, V., Fehl, N. y Poston, W. (2012). Mission essential fitness: comparison of functional circuit training to traditional Army physical training for active duty military. Military Medicine, 177(10), 1125-1130. DOI: https:// doi.org/10.7205/milmed-d-12-00143

Hernández Sampiere, R. (2010). Metodología de la investigación. 2da. edición. México D. F., México: Editorial Ultra.

Hernández, H. (2000). Selección de talentos para la iniciación deportiva, una experiencia cubana. ISCF Universidad Manuel Fajardo, La Habana, Cuba.

Hollingsworth, D. (2009). The prevalence and impact of musculoskeletal injuries during a pre-deployment workup cycle: survey of a Marine Corps special operations company. Journal of Special Operations Medicine, 5-11. Recuperado de https://www.ncbi.nlm.nih.gov/pubmed/20112642

Hong, S. y Chu, I. (2009). Stress fracture of the proximal fibula in military recruits. Clin Orthop Surg, 161-164. DOI: https://doi.org/10.4055/cios.2009.1.3.161

Keith, H., Bruce, J., Steven, H. y Bullock, D. (2010). Musculoskeletal injuries description of an under-recognized injury problem among military personnel. American Journal of Preventive Medicine, 61-70. DOI: https://doi. org/10.1016/j.amepre.2009.10.021

Martin, D. (2001). Manual de metodología del entrenamiento deportivo. Barcelona, España: Paidotribo.

Martínez, J. (2011). Aspectos psicológicos de la supervivencia en operaciones militares. Sanidad Militar, 67(1), 43-48. DOI: https://doi.org/10.4321/ s1887-85712011000100009

Mohammadi, F., Azma, K., Naseh, I., Emadifard, R. y Etemadi, Y. (2013). Military exercises, Knee and ankle joint position sense, and injury in male cons- 
cripts: A Pilot Study. Athletic Training Education Journal. DOI: https://doi. org/10.4085/1062-6050-48.3.06

Olmedilla-Zafra, A., Ortega-Toro, E., Prieto-Andréu, J. y Redondo, A. (2009). Percepción de los tenistas respecto a los factores que pueden provocar lesiones: diferencias entre federados y no federados. Cuadernos de Psicología del Deporte, 9(2), 7-18. DOI: https://doi.org/10.4321/s1578-84232013000100009

Rodríguez Gómez, J., Valenzuela Pinzón, J., Velasco Rodríguez, J., Castro Jiménez, L. (2016). Caracterización de las lesiones derivadas del entrenamiento físico militar. Revista Cuidarte, 7(1), 1219-1226. DOI: https://doi.org/10.15649/cuidarte.v7i1.301

Weineck, J. (2005). Entrenamiento total. Barcelona, España: Paidotribo. 